\title{
Variable Latency Approach in VLSI Adder Implemented to Reduce Area and Power
}

\author{
K. Kaarthik and C. Vivek \\ Department of Electronics and Communication Engineering, \\ M. Kumarasamy College of Engineering (Autonomous), Thalavapalayam, Karur - 639113, Tamil Nadu, India; \\ kaarthikk.ece@mkce.ac.in, vivekc.ece@mkce.ac.in
}

\begin{abstract}
Objective: The Ultimate aim of the VLSI Design is to improve the efficiency, Reduction of Delay and Power Consumption and to minimize the area. In our proposed approach we had implemented the analysis had been done on the field of Speed, Power consumption, Area and Power delay product (PDP) for a carry skip adder with other adders listed as the parallel prefix adders and others. Methods/Statistical Analysis: The Carry-Skip Adder planned here reduces the time required to propagate the carry by skipping over teams of consecutive adder stages, is understood to be comparable in speed to the carry look-ahead technique whereas it uses less logic space and fewer power. Findings: The adders are basic building blocks of the digital circuits for the Signal processing, Integrating and other process of operation. There are various types of adders are proposed in Literature which are commonly used in VLSI Design. The Simulation results also shows that the proposed adder Architecture is Faster and Area efficient compared to other existing adder architecture. Application/ Improvements: They estimate the performance of proposed design will be better in terms of Logic and route delay by experimental results.
\end{abstract}

Keywords: Adders, Carry Bypass Adder (CBA), Carry Increment Adder (CIA), Carry Look-Ahead Adder (CLA), Carry Skip Adder (CSkA), Han Carlson Adder (HCA), Ripple Carry Adder (RCA)

\section{Introduction}

Adders measure the building hinder in DSP applications. The key component in DSP is that the parallel adder and furthermore basic approach of the parallel tree base approach is allowed in the full adder cell structure. The Full Adder architecture can produce two outputs on considering the three inputs - add up to $S$ and Carry Cout. By falling the total adder cells, we've the key Adder structure in which "N" full adder cells square measure fell to ask the $\mathrm{n}$ - bit RCA and convey created at ordinal piece is given on the grounds that the contribution to the $n+1$ bit furthermore to the A and B inputs ${ }^{1,2}$. As Ripple Carry Adder is that the most straightforward one among the inverse adders however moreover slowest of every one of them, thus of the spread of the convey from LSB to shared reserve funds bank. Viper Structures separated from adder square measure CLA, CSA, CSLA, CSKA and so on. Ripple carry adder is slowest among all the viper structure accordingly of the wavelet of the convey. Convey look Ahead viper tackles the matter of convey spread from ideal to left by exploitation convey proliferate and furthermore the convey create signals for diminishing postponement however the world, assortment of entryways is improved thus nature of the framework is upgraded. In Carry skip viper, the spread time of convey is diminished by skirting the group of adder stages and introduces equipment and execution compromise ${ }^{2,3}$.

Efficient Full Adder Some of the quality economical full adders square measure compared and also the full adder with less power is taken into account for the look of RCA and 3 stages of CSA. There square measure differing types of CMOS full adder. This section reviewed the 3 progressive 1-bit full adders. This proposed cell is

*Author for correspondence 
compared with them. The bridge has twenty six transistors this style creates a conditional conjunction between 2 circuit nodes. Full Adders that square measure supported totally trigonal CMOS vogue square measure referred to as Bridge Full Adders. The Full-Adder with twenty four Transistors (FA24T) has twenty four transistors this full Adder relies on Bridge vogue.

\section{Carry Skip Adder}

In our VLSI Design adders there were several architectures were designed to attain the Increase in Speed and Area in the VLSI Adder Architecture. The Architecture is to achieve the expected output as increase in speed, Area reduction and Power consumption of the Architecture has to be reduced.

A Carry Skip Adder (likewise alluded to as a convey sidestep viper) is a viper execution that enhances the deferral of a Ripple carry viper with almost no exertion contrasted with option adders. The progress of the most pessimistic scenario deferral is accomplished by exploitation many convey skip adders to make a piece convey skip adder $^{3-5}$.

The CSKA that is a save adder similarly as power use and Area utilize. The fundamental path delay of the CSKA is far tinier than the one inside the RCA, while its space and power usage square measure similar to those of the RCA. Likewise, the power-concede thing (PDP) of the CSKA is smaller than those of the CSLA and PPA structures. Besides, because of the little extent of transistors, the CSKA purposes of enthusiasm from almost short wiring lengths correspondingly as a step by step and direct configuration. The moderately bring down speed of this adder structure, be that as it may, limits its utilization for fast applications. Amid this paper, given the connecting with alternatives of the CSKA structure, we have focused on diminishing its deferral by changing its execution bolstered the static CMOS rationale. The focus on the static CMOS begins from the will to have a constantly in operation circuit underneath a decent fluctuate of give voltages in to a great degree scaled technologies ${ }^{6}$. The arranged change will build the speed altogether though keeping up the low space and power utilization alternatives of the CSKA. Also, A conformity of the structure, upheld the variable inertness technique, that logically cuts down the workplace use while not basically influencing the CSKA speed, is also introduced. To the best of our information, no work concentrating on style of CSKAs in operation from the super edge zone specifically down to close edge region and conjointly, the orchestrating of (cream) variable lethargy CSKA structures are represented inside the written work. From now on, the responsibilities of this paper are oftentimes compacted as follows ${ }^{7,8}$ proposing a changed CSKA structure by consolidating the link and hence the incrimination plans to the conventional CSKA (Conv-CSKA) structure for upgrading the speed and vitality strength of the adder. The adjustment furnishes North American nation with the adaptability to utilize less convoluted convey skip rationales upheld the AOI/OAI compound doors as opposed to the electronic device?.

- Providing a style system for creating Associate in nursing mild CSKA structure maintained coherently expressions introduced for the imperative way delay ${ }^{10,11}$.

- Examining the impact of voltage scaling on the quality of the masterminded CSKA structure (from the apparent offer voltage to the nearby edge voltage).

- Proposing a cross breed variable idleness CSKA structure reinforced the development of the showed CSKA, by substitution a portion of within stages in its structure with a PPA that is changed in the midst of this paper ${ }^{12}$.

The straggling leftover of this paper is dealt with as takes after. It discusses related manage changing the CSKA structure for up the speed likewise as past work that usage variable dormancy structures for growing the force of adders at low offer voltages. The Conv-CSKA as in Figure 1 with mounted stage appraise (FSS) and variable

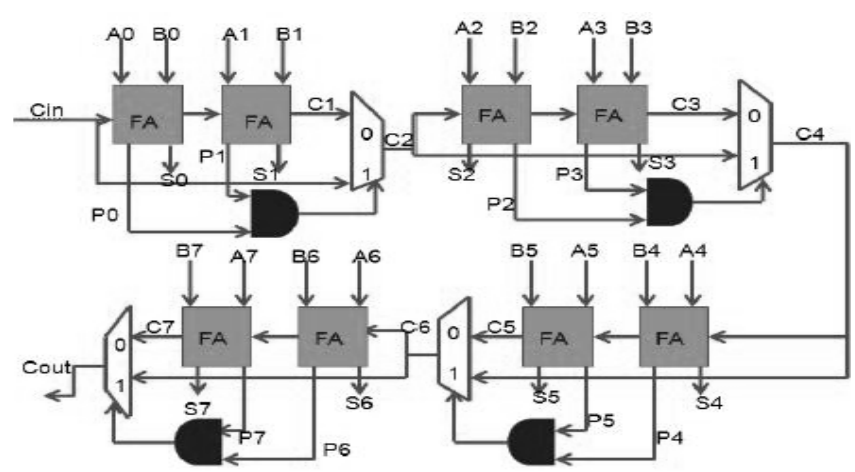

Figure 1. Carry Skip Adder. 
stage measure (VSS) is cleared up, while it portrays the expected static CSKA structure. The cross breed variable inaction CSKA structure is regularly endorsed in it. The eventual outcomes of examination the properties of the foreseen structures with those of choice adders ${ }^{13}$.

\section{Conventional Method}

The standard structure of the CSKA includes stages containing chain of full adders (FAs) (RCA square) and 2:1 electronic contraption (pass on skip method of reasoning). The RCA pieces square measure related with every choice through 2:1 multiplexers, which may be set into one or additional level structures. The CSKA design (i.e., the measure of the FAs per organize) incorporates a decent effect on the speed of this sort of viper a few methodologies are encouraged for finding the ideal scope of the FAs. The strategies gave in make utilization of VSSs to weaken the deferral of adders bolstered a solitary level convey skip rationale. A few systems to develop the speed of the development CSKAs square measure arranged. The procedures, nonetheless, cause space and power increment fundamentally and less general format. the look of a static CMOS CSKA wherever the phases of the CSKA have a variable sizes. Furthermore, to bring down the spread postponement of the adder, in each stage, the convey look-ahead rationales were utilized. Once more, it had a favor design in addition as monstrous power utilization and space use. Also, the look approach, that was presented only for the 32-bit adder, wasn't general to be connected for structures with entirely unexpected bits lengths ${ }^{14,15}$.

Alioto and Palumbo propose a direct system for the look of a solitary level CSKA. the strategy is predicated on the VSS method wherever the close ideal quantities of the FAs square measure decided upheld the skip time (postponement of the multiplexer), and furthermore the swell time (the time required by a help to swell through a FA). The objective of this technique is to diminish the basic way delay by considering a non entire number quantitative connection of the skip time to the swell time on in opposition to a large portion of the past works, that contemplated Associate in Nursing entire number quantitative connection. through and through of the works surveyed to date, the primary target was on the speed, while the office utilization and space use of the CSKAs weren't contemplated as in Figure 2. Notwithstanding for the speed, the deferral of skip rationales, that square measure bolstered multiplexers and kind a curiously large a piece of the viper fundamental way delay, has not been reduced $^{16,17}$.

\section{Results and Discussion}

The need to downsize the capacity utilization is to help the speed and to upgrade the intensity of the conventional convey skip viper. We need to downsize the capacity utilization of the look while not affecting the speed of it and even need to reduce the world and requesting way delay. In existing procedure inferable from the utilization of electronic gadget that goes about as skip rationale may winds up in an outsized basic way deferral and space use and furthermore outsized power utilization. These downsides are coming to be unraveled inside the arranged style by exploitation compound doors instead of electronic gadget.

We exploit the electronic gadget rationale by which the amount of entryway tally is extra. The Ripple carry

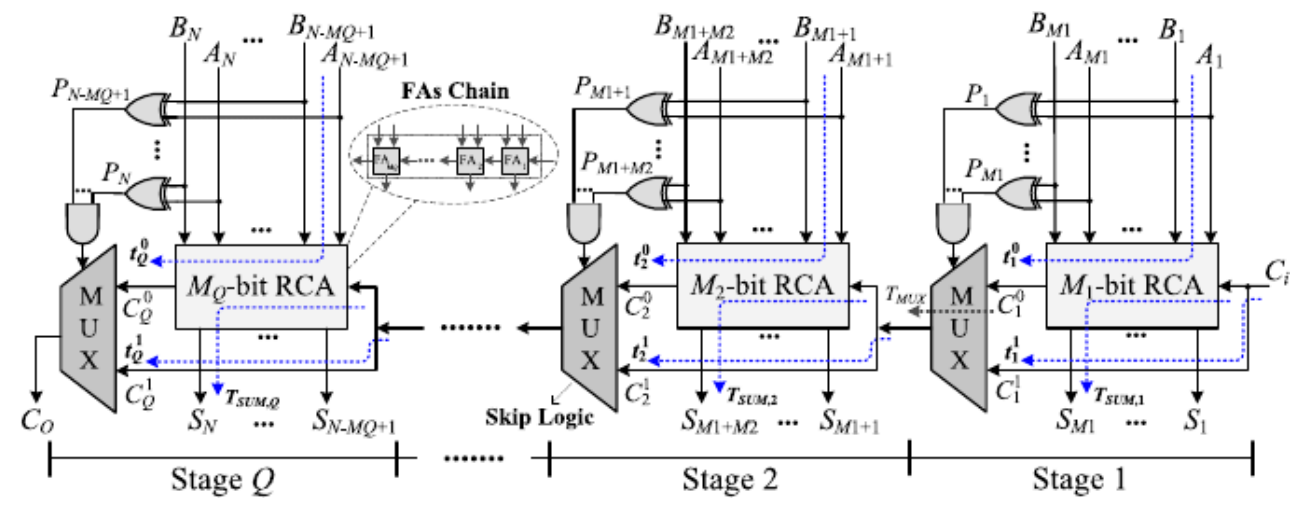

Figure 2. Conventional structure of the CSKA. 
adder is made by falling full adders (FA) squares serial. One full adder is responsible for the expansion of 2 paired digits at any phase of the swell carry ${ }^{18-20}$.

Various full adders might be added to the Ripple carry adder or Ripple carry adders of various sizes might be fallen keeping in mind the end goal to oblige twofold vectors strings of bigger sizes. For an $\mathrm{n}$ bit parallel adder it requires $\mathrm{n}$ computational components. The convey is proliferated in a serial calculation. Consequently, a deferral is more as the quantity of bits is expanded in $\mathrm{RCA}^{21-23}$.

\section{Proposed Method}

We tested all of our components using simulation through the modelsim 6.2, the area, power and delay of the various adders are been compared and obtained the outputs in the form of chart. In this obtained output the power distribution of the Carry Skip Adder is higher when compared with the all other adders which could be highly efficient for an IC chip to provide the results. The delay is also a major term to be concerned while the execution of results, if the delay is higher, then the processing speed will be noticed as less even it is an efficient adder the delay is higher it is not been considered. The delay is also a major factor; in Carry Skip Adder it lowers when compared with the other types of adders which could be a major advantage for our adder ${ }^{24-25}$.

In Carry Skip Adder there are two types: Fixed Carry Skip Adder and Variable Carry Skip Adder. The Variable Carry Skip Adder provides better results as low power, Reduced Delay, High PDP and small area so it can be identified as better type from ${ }^{26}$ to provide the Best result (Figure 3).

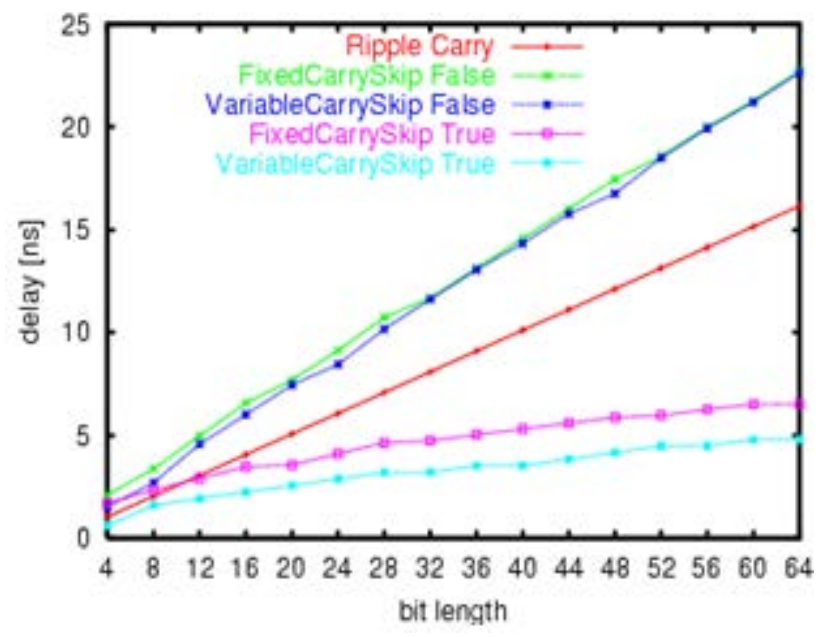

Figure 3. Comparison Graph of Various Carry Skip Adder.

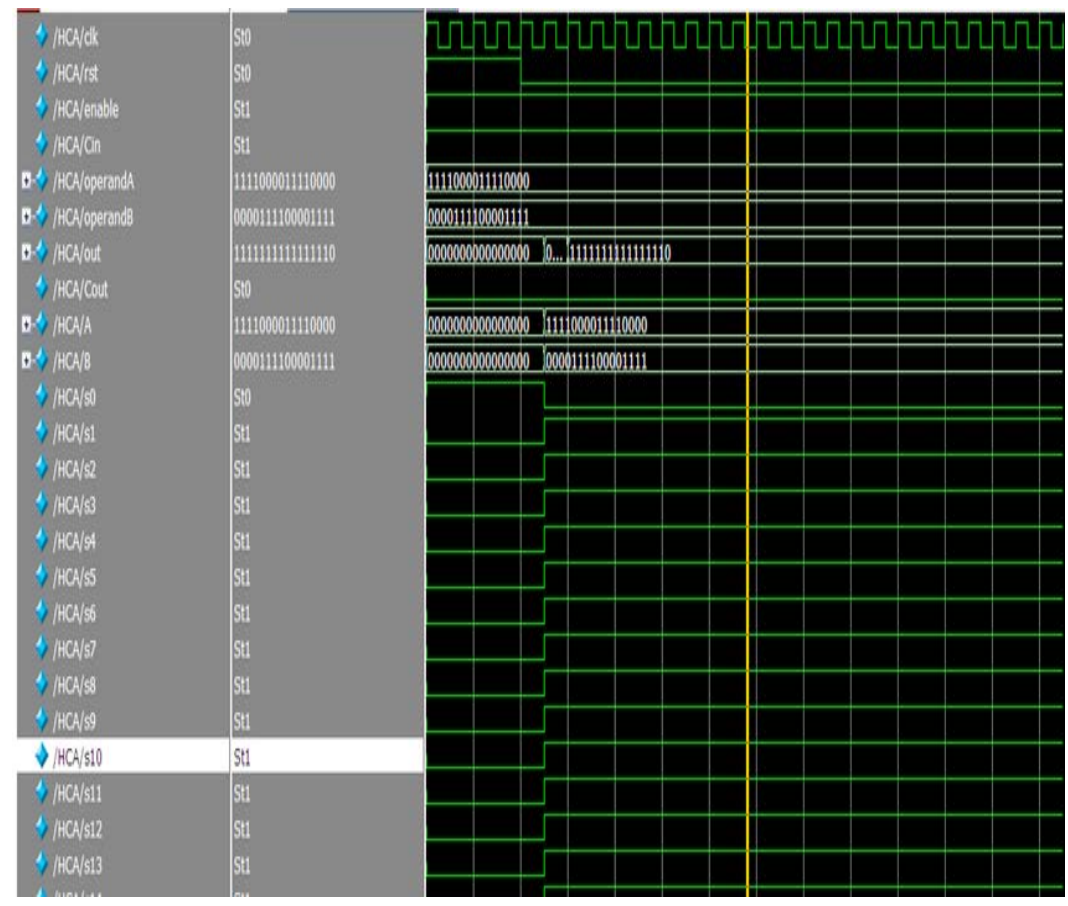

Figure 4. Schematics of Han Carlson Adder in ModelSim tool. 


\section{Simulation Results}

\subsection{Han Carlson Adder}

The Schematic representation of Simulation result shows the output of the Han Carlson Adder. In that result the ModelSim-Altera 6.4a (Quartus II 9.0) Starter Edition represents the Area and delay of the Han Carlson Adder (Figure 4). The Quartus II 9.0 Web Edition simulation result shows the Power dissipation value obtained (Figure 5) which includes the Thermal Power dissipation of both Static and Dynamic is $62.13 \mathrm{~mW}, 1.46 \mathrm{~mW}, 46.12$ $\mathrm{mW}$ and Power estimation is used to provide weather the output is low or high and the Power estimation of Han Carlson Adder is Low. But it can able to provide the exact output most of the time, not always. So an error detection and correction circuit is essential for the Han Carlson Adder.

\begin{tabular}{|c|c|}
\hline PowerPlay Power Analyzer Summary & \\
\hline PowerPlay Power Anslyzer Slatus & Succestul \\
\hline Quatus II Version & 9.0 buld $13202 / 25 / 2009$ SJ Web Edtion \\
\hline Revision Name & hea \\
\hline Toptevel Ertty Name & $\mathrm{HCA}$ \\
\hline Fanly & Cyolone III \\
\hline Device & EP3C5F256C6 \\
\hline Power Models & Final \\
\hline Total Themal Power Dissipation & $62.13 \mathrm{~mW}$ \\
\hline Core Dynamic Themal Power Dissipation & $1.46 \mathrm{~mW}$ \\
\hline Core Sratic Themal Power Dissipation & $46.12 \mathrm{~mW}$ \\
\hline VO Themal Power Dissipation & $14.54 \mathrm{~mW}$ \\
\hline Power Estimation Corfidence & Low: user provided inaufficient togple rate data \\
\hline
\end{tabular}

Figure 5. Schematics of Han Carlson Adder Quartus II Power analyzer.

\subsection{Carry Skip Adder}

The Schematic represented Simulation result shows the output of the Carry Skip Adder. In that result the ModelSim-Altera 6.4a (Quartus II 9.0) Starter Edition which represents the Area and delay of the Carry Skip Adder (Figure 6). The Quartus II 9.0 Web Edition simulation result shows the Power dissipation value (Figure 7). It includes the Thermal Power dissipation of both Static and Dynamic which are $48.00 \mathrm{~mW}, 1.18 \mathrm{~mW}$ and $48.00 \mathrm{~mW}$. The Power estimation is used to provide weather the output is low or high and the Power estimation of Carry Skip Adder is Low when compared to the Han Carlson Adder.

\begin{tabular}{|ll|}
\hline PowerPlay Power Analyzer Summary & \\
\hline PowerPlay Power Analyzer Status & Successful \\
Quatus II Version & 9.0 Buld 132 02/25/2009 SJ Web Edtion \\
Revision Name & NbtCanySkipAdder \\
Toptevel Entty Name & NbtCanySkipAdder \\
Family & Cyclone \\
Device & EP1C3T144C6 \\
Power Models & Final \\
Total Themal Power Dissipation & $48.00 \mathrm{~mW}$ \\
Core Dynamic Themal Power Dissipation & $0.00 \mathrm{~mW}$ \\
Core Static Themal Power Dissipation & $48.00 \mathrm{~mW}$ \\
IO Thermal Power Dissipation & $0.00 \mathrm{~mW}$ \\
Power Estimation Corfidence & Low: user provided insufficient toggle rate data \\
\hline
\end{tabular}

Figure 7. Schematics of Carry Skip Adder Quartus II Power analyzer.

The various parameters such as Area, Power, and Delay are analyzed and listed in Table 1 and their outputs are considered. From their output a chart has been obtained which provides clear information about Performance of Various adders (Figure 8). The Delay of the Carry Skip

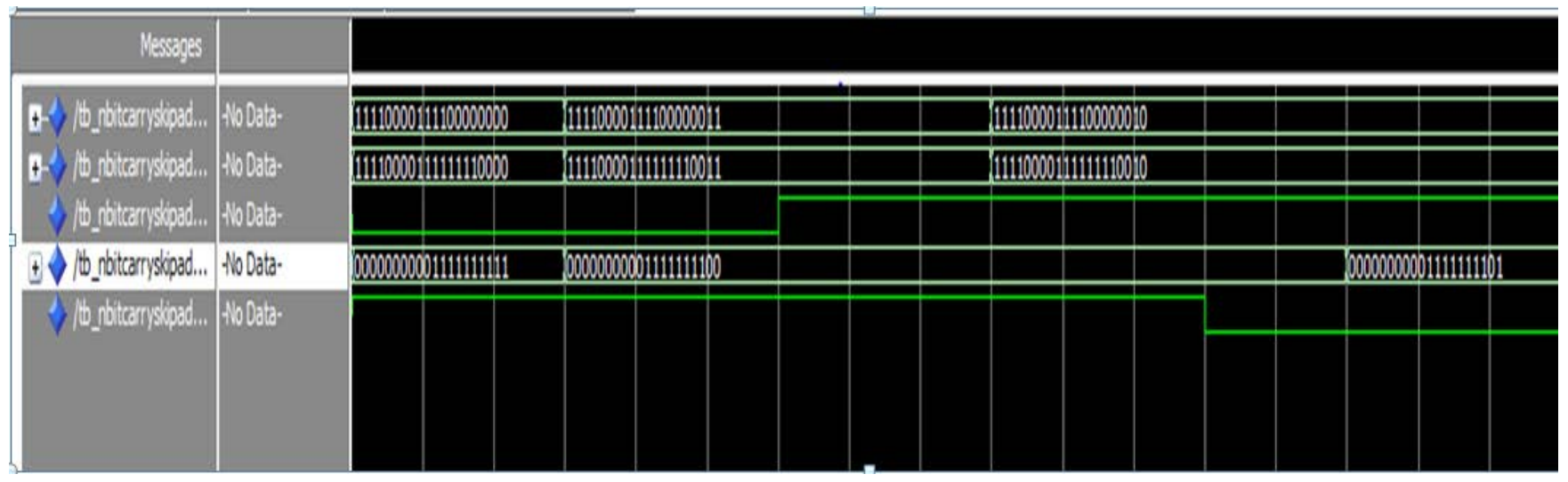

Figure 6. Schematics of Carry Skip Adder in ModelSim tool. 
Adder is less when compared with others, then if Delay is low the Power consumption will be low and the output can be obtained with short span of time. So the Carry Skip Adder can be a right choice for the VLSI Architecture.

From the above performance analysis (Figure 9) it is observed that, RCA, CIA and CSkA are having better performance in terms of area (LUT's and Slices). In those adders particularly the Carry Skip Adder is having better area reduction factor which might be considered.

\section{Conclusion}

The Carry-Skip Adder (CSA) planned here reduces the time required to propagate the carry by skipping over teams of consecutive adder stages which is understood to be comparable in speed to the carry look-ahead technique; it uses less logic space and fewer power. Thus, the simulation results shows that CSA is quicker than Normal Adders and Parallel Prefix Adders.

Table 1. Performance Analysis Table of Various Adders

\begin{tabular}{|l|l|l|l|}
\hline S. No. & Power (in $\mathrm{mW})$ Distribution & Gate Count & Delay (in nS) \\
\hline RCA & 0.206 & 288 & 4.208 \\
\hline CSKA & 0.603 & 396 & 1.623 \\
\hline CLA & 0.312 & 272 & 3.1 \\
\hline CIA & 0.261 & 342 & 2.83 \\
\hline CBA & 0.459 & 266 & 3.01 \\
\hline HC & 0.579 & 360 & 2.262 \\
\hline
\end{tabular}

\section{Performance Analysis of Various Adders}

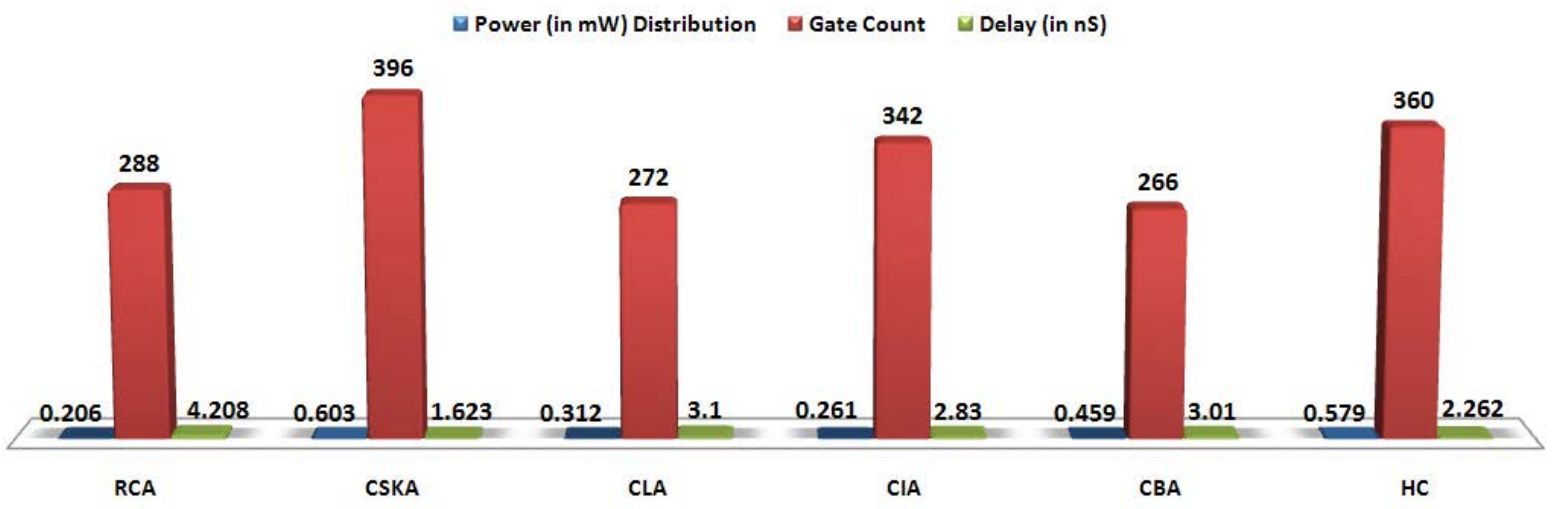

Figure 8. Performance Analysis Chart of Various Adders.

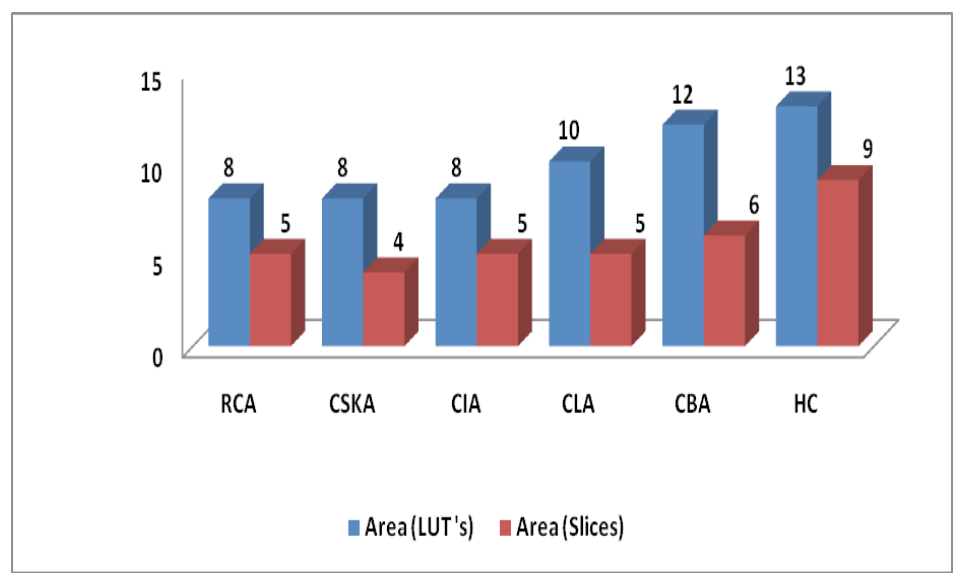

Figure 9. Performance Analysis Chart of Area on Various Adders. 


\section{References}

1. Tamar HG, Tamar AG, Hadidi K, Khoei A, Hoseini P. High Speed Area Reduced 64-bit Static Hybrid CarryLookahead/Carry-Select Adder. 18th IEEE International Conference on Electronics, Circuits, and Systems. 2011; p. 460-3. crossref.

2. Kaur J, Sood L. Comparison Between Various Types of Adder Topologies. International Journal of Computer Science and Technology. 2015; 6(1):1-5.

3. Uma R, Vijayan V, Mohanapriya M, Paul S. Area, Delay and Power Comparison of Adder Topologies. International Journal of VLSI design \& Communication Systems (VLSICS). 2012; 3(1):1-16.

4. Padmajarani SV, Muralidhar M. A Hybrid Parallel Prefix Adder for high speed computing. Proceedings 7 National Conference on Advances in Electronics and Communications (ADELCOs). National Engineering College, Kovilpatti, India. 2011.

5. Singh S, Kumar D. Design of area and power efficient modified carry select adder. International Journal of Computer Applications. 2011; 33(3):1-5.

6. Shirakol SK, Kulkarni AS, Akash AF, Amminabhavi NN, Parvati A. Design and Implementation of 16-Bit Carry Skip Adder using Efficient Low Power High Performance Full Adders. Conference: Second International Conference on Emerging Research in Computing, Information, Communication and Application ERCICA. 2014 Aug; 2:1-10.

7. Mondal SR, Bhowmik M, Maity S, Sultana R. Comparative Analysis and Study on 4-bit RCA and CSK using CMOS Logic. International Journal of Soft Computing and Engineering. 2015 January; 4(6):2231-307.

8. Swapna K. Gedam and Pravin P. Zode. Parallel Prefix Han-Carlson Adder. International Journal of Research in Engineering and Applied Sciences. 2014; 2(2):1-4.

9. Choi V, Swartz Lander EE. Parallel Prefix adder design with matrix representation. Proceedings, 17th IEEE Symposium on Computer Arithmetic (ARITH'05). 2005; p 90-8. PMid:15647877.

10. Sun Y, Zhang X, Jin X. High-Performance Carry Select Adder Using Fast All-one Finding Logic. AICMS, Second Asia International Conference, Modeling \& Simulation. 2008 May; p. 1012-14. PMid:18163628.

11. Beaumont-Smith A, Lim C. Parallel prefix adder design. Proceedings, 15th IEEE Symposium on Computer Arithmetic. ARITH-15. 2001 June; p. 218-25. crossref.

12. Vivek C, Rajan SP, Kavitha V. Implementation of High Speed Area Efficient Variable Latency Adder. Asian Journal of Research in Social Sciences and Humanities. 2016 Sep; 6(9):306-16. crossref.
13. Kaarthik K, Vivek C. Hybrid Han Carlson Adder Architecture for Reducing Power and Delay. Middle East Journal of Scientific Research. 2016; 24:308-13.

14. Vitoroulis K, Al-Khalili AJ. Performance of Parallel Prefix Adders Implemented with FPGA technology. IEEE Northeast Workshop on Circuits and Systems. 2007; p. 498501. crossref.

15. Kaarthik K, Vivek C. A survey on Han-Carlson adder with efficient adders. International Journal of Emerging Technology in Computer Science \& Electronics (IJETCSE). 2016 Feb; 20(2):81-7.

16. Kaarthik K, Pradeep S, Selvi S. An Efficient Architecture Implemented to Reduce Area in VLSI Adders. Imperial Journal of Interdisciplinary Research (IJIR). 2017; 3(2):326-30.

17. Dao H, Oklobdzija VG. Application of logical effort techniques for speed optimization and analysis of representative adders. Conference Record of Thirty-Fifth Asilomar Conference on Signals, Systems and Computers (Cat. No.01CH37256). 2001; 2:1666-9.

18. Vishwaja S, Kaarthi K. VLSI Architecture Using Expanded Hyperbolic CORDIC Algorithm. International Journal of Applied Engineering Research (IJAER). 2015; 10(33):26051-4.

19. Vivek C, Rajan SP. Z-TCAM: An Efficient Memory Architecture Based TCAM. Asian Journal of Information Technology. 2016; 15(3):448-54.

20. Arulpriya S, Davood KS. Analysis of MLSA for Low Power CAM Design. Journal of Chemical and Pharmaceutical Sciences. 2016; 12(8):104-07.

21. Rajarajachozhan C, Daniel Raj A, Deb S. Compensating Threshold Voltage Roll-Off in Nanoscale MOSFET with Parameter Adjustment. Journal of VLSI Design and Signal Processing. 2015; 1(2):1-7.

22. Vivek C, Rajan SP. Review of Low Power and High Speed Implementation of 3-bit Flash Analog to Digital Converter. Journal of Chemical and Pharmaceutical Sciences. 2016; 8:74-8.

23. Iyer A, Kumar PH, Jayasurya P, Karpagam M, Kaarthik K. A Comparative Survey on Carry Skip adder with other Efficient Adders. Journal of Chemical and Pharmaceutical Sciences. 2017; 4:116-21.

24. Dimitrakopoulos G, Nikolos D. High speed parallel prefix VLSI Ling adders. IEEE Transactions on Computers. 2005 Feb; 54(2):225-31. crossref.

25. Ziegler MM, Stan MR. A unified design space for regular parallel prefix adders. Proceedings Design, Automation and Test in Europe Conference and Exhibition. 2004; 2:1386-7. crossref.

26. Vivek C, Rajan SP. Design of Data Aware Low Power Area Efficient Data paths for Processing Elements in a Reconfigurable System. International Journal of Computer Science and Information Security. 2016; 14(9):1100-13. 